\title{
The Scaling Limit and Osterwalder-Schrader Axioms for the Two-Dimensional Ising Model
}

\author{
Ricardo Schor and Michael O'Carroll \\ Departamento de Fisica do ICEx, Universidade Federal de Minas Gerais, Belo Horizonte, \\ Minas Gerais. Brasil
}

\begin{abstract}
From a Feynman-Kac formula in a Fermion Fock space for the Schwinger functions of the infinite lattice periodic two-dimensional Ising model, scaled and scaling limit Schwinger functions are defined and shown to admit an absolutely convergent series representation. As the critical temperature is attained, it is shown that the scaled Schwinger functions converge and that the resulting scaling limit Schwinger functions obey the Osterwalder-Schrader axioms.
\end{abstract}

\section{Introduction}

In [1] the transfer matrix for the two-dimensional finite periodic lattice Ising model was diagonalized in terms of finite lattice Fermions. In [2], starting from a finite lattice Feynman-Kac $(\mathrm{F}-\mathrm{K})$ formula, series representations for infinite lattice correlation functions were defined. In [3] we showed that the $k$-point infinite lattice correlation functions $S_{k}$ are represented by a $\mathrm{F}-\mathrm{K}$ formula in a Fermion Fock space. In this representation two sets of canonical Fermion operators, related by a proper linear canonical transformation (plct), are utilized (see [4]) and energymomentum and spin operators are defined. In [5] a generalization of Wick's theorem was proved for plct and used to obtain explicit series representations for $S_{k}$. We also defined series representations for scaling limit Schwinger functions $S_{k}^{L}$ from above $\left(T^{+}\right)$and below $\left(T^{-}\right)$the critical temperature $T_{c}$. The $S_{k}^{L}$ are natural candidates for the Schwinger functions of a Wightman field theory.

In this article we show that the $S_{k}^{L}$ are the limits of scaled infinite lattice Schwinger functions and that the $S_{k}^{L}$ satisfy the Osterwalder-Schrader $(\mathrm{O}-\mathrm{S})$ axioms [7].

In Sect. II we introduce scaled Schwinger functions $S_{k \lambda}=S_{k}(\lambda) / Z_{k \lambda}$, where $\lambda \in[0,1]$ is a scaling parameter that depends on the temperature $T ; \lambda \rightarrow 0$ as $T \rightarrow T_{c}$, and $Z_{k \lambda}$ is a wave function renormalization. We prove absolute convergence of the series representation for $S_{k \lambda}$, uniform in $\lambda$, as well as convergence to the scaling limit, i.e. $\lim _{\lambda \rightarrow 0} S_{k \lambda}=S_{k}^{L}$. From these results the series for $S_{k}^{L}$ manifestly 
satisfies Osterwalder-Schrader $(\mathrm{O}-\mathrm{S})$ positivity; O-S symmetry also follows.

In Sect. III a factorization theorem for the scaling limit of Fermion matrix elements of the spin operator is proved and used to obtain strict upper and lower bounds on $S_{2}^{L}$ which imply O-S temperedness. $S_{2}^{L}$ is less singular than $R^{-1 / \pi}$, for small $R$, where $R$ is the Euclidean distance. Bounds on $S_{k}^{L}, k>2$, which imply $\mathrm{O}-\mathrm{S}$ temperedness are obtained by combining the existence of the scaling limit with bounds on $S_{2}^{L}$ and using a result of [6]. For $T^{+}$a clustering property is proved which implies the uniqueness of the vacuum of the reconstructed Wightman theory.

Formally, Poincare invariance of the real time Schwinger functions, the Wightman distributions, is seen most easily using rapidity variables. In these variables we give a simple proof of rotational invariance of $S_{2}^{L}$ in Sect. IV; for $k>2$ a more technical proof is needed and is given in Appendix D.

A key ingredient in showing the convergence of the series for $S_{k \lambda}$ is a combinatorial lemma for the expansion of Pfaffians which we give in Appendix A. In Appendix B bounds and limits of various scaling functions that occur in $S_{k \lambda}$ are obtained. In addition to the results of Appendix $A$, in order to prove convergence of the scaled $S_{k \lambda}$ and rotational invariance of $S_{k}^{L}, k>2$, we use properties of scaled, Hilbert transforms and rapidity transforms given in Appendix C.

For other approaches to the scaling limit see [2-II], [8] and [9].

\section{Scaling Limit}

We define scaled Schwinger functions, $S_{k \lambda}, k=0,1, \ldots \infty, \lambda \in[0,1]$, by

$$
S_{k \lambda}\left(s_{1}^{\prime}, x_{1}^{\prime}, \ldots, s_{k}^{\prime}, x_{k}^{\prime}\right)=S_{k}\left(s_{1}^{\prime} / \lambda, x_{1}^{\prime} / \lambda, \ldots, s_{k}^{\prime} / \lambda, x_{k}^{\prime} / \lambda ; K(\lambda)\right) / Z_{k \lambda}
$$

for $\lambda \in(0,1], s_{1}^{\prime}, x_{1}^{\prime} \in R, 1 \leqq i \leqq k, s_{1}^{\prime}<s_{2}^{\prime}, \ldots,<s_{k}^{\prime}$, where $K(\lambda)=J T(\lambda)^{-1}, J>0, T$ the temperature, and $K(\lambda) \rightarrow K_{c}$ (critical coupling) as $\lambda \rightarrow 0$ (see Appendix B). $S_{k}$ are the infinite lattice Schwinger functions given by the Feynman-Kac formula of [3] extended to the continuous $s_{1}^{\prime}, x_{i}^{\prime}$. For $T>T_{c} Z_{k \lambda}=\left(x_{1} x_{2}\right)^{k / 2}$. $((\mid 1-$ $\left.\left.\left.\sinh ^{2} 2 K\right|^{2}\right)^{1 / 8} / \cosh K^{*}\right)^{k}, \tanh K^{*}=e^{-2 K}$. For $\lambda=0$ set $S_{k 0} \equiv S_{k}^{L}$, the scaling limit Schwinger functions defined in [5]. From the series representation for $S_{k}$ for $T>T_{c}$ in [5] we have the following representation for $S_{2 N \lambda}, \lambda \in(0,1]$, in the difference variables, denoted by $\left\{s_{i}, x_{i}\right\}_{i=1}^{2 N-1}$ :

$S_{2 N \lambda}=\sum_{\left\{m^{2 k-1}\right\}_{1}^{N} \operatorname{Odd}\{m 2 k\}_{1}^{N} \text { even }} \prod_{l=2 N-1}^{1} T_{m_{l+1}, m_{l}}^{\lambda}\left(x_{l+1}, s_{l+1} ; x_{l}, s_{l}\right) \theta_{m_{1}}^{\lambda}\left(x_{1}, s_{1}\right)$,

where $T_{m_{2 N} m_{2 N-1}}\left(x_{2 N}, s_{2 N} ; x_{2 N-1}, s_{2 N-1}\right) \equiv T_{m_{2 N-1}}\left(x_{2 N-1}, s_{2 N-1}\right)$ and the linear operators $T_{m_{j} m_{i}}^{\lambda}\left(x_{j}, s_{j}: x_{i}, s_{i}\right) \equiv T_{j i}^{\lambda}: L^{2}\left(R^{m_{i}}\right) \rightarrow L^{2}\left(R^{m_{j}}\right)$ and $T_{m_{i}}^{\lambda}\left(x_{i}, s_{i}\right) \equiv T_{i}^{\lambda}: L^{2}\left(R^{m_{i}}\right) \rightarrow C$, $\lambda \in[0,1]$, are defined as

$$
\begin{aligned}
\left(T_{j i}^{\lambda} f\right)\left(\left(p^{j}\right)_{1, m_{j}}\right)= & \int \ldots \int L_{\lambda}^{x}\left(\left(-p^{j}\right)_{1, m_{j}} \mid\left(p^{i}\right)_{1, m_{i}}\right) \\
& \cdot \exp \left\{-\frac{1}{2} \sum_{k=i, j} \sum_{l=1}^{m_{k}}\left(\omega_{\lambda}\left(p_{l}^{k}\right) s_{k}+i p_{l}^{k} x_{k}\right)\right\} \\
& \cdot f\left(\left(p^{i}\right)_{1, m_{i}}\right) d^{m_{i}} p^{i}, j>0 \text { even, } i \text { odd }
\end{aligned}
$$




$$
\begin{aligned}
\left(T_{i j}^{\lambda} f\right)\left(\left(p^{i}\right)_{1, m_{i}}\right)= & \left.\int L_{\lambda}^{x}\left(-p^{j}\right)_{1, m_{j}} \mid\left(p^{i}\right)_{1, m_{i}}\right) \\
& \cdot \exp \left\{-\frac{1}{2} \sum_{k=i, j} \sum_{l=1}^{m_{k}}\left(\omega_{\lambda}\left(p_{l}^{k}\right) s_{k}+i p_{l}^{k} x_{k}\right)\right\} \\
& \cdot f\left(\left(p^{j}\right)_{1, m_{J}}\right) d^{m_{J}} p^{j}, j>0 \text { even, } i \text { odd } ; \\
T_{i}^{\lambda} f= & \int L_{\lambda}^{x}\left(\phi \mid\left(p^{i}\right)_{1, m_{i}}\right) \exp \left\{-\frac{1}{2} \sum_{l=1}^{m_{i}}\left(\omega_{\lambda}\left(p_{l}^{i}\right) s_{i}+i p_{l}^{i} x_{i}\right)\right\} \\
& \cdot f\left(\left(p^{i}\right)_{1, m_{l}}\right) d^{m_{l}} p^{i}, i \text { odd }
\end{aligned}
$$

for $\lambda \in(0,1]$. Also define $\theta_{m_{1}}^{\lambda}\left(s_{1}, x_{1}\right) \equiv \theta_{1}^{\lambda}$ by

$$
\theta_{1}^{\lambda}\left((p)_{1, m_{1}}\right)=\overline{L_{\lambda}^{x}\left(\phi \mid(p)_{1, m_{1}}\right)} \exp \left\{-\frac{1}{2} \sum_{l=1}^{m_{1}}\left(\omega_{\lambda}\left(p_{l}\right) s_{1}+i p_{l} x_{1}\right)\right\} .
$$

For $\lambda=0$ define $T_{j i}^{0} \equiv T_{j i}, T_{i}^{0}=T_{i}$ and $\theta_{1}^{0}=\theta_{1}$ by using the $\lambda \rightarrow 0$ limit functions of Appendix B.

In the above $\omega_{\lambda}(p)=\varepsilon(\lambda p) / \lambda, \quad \cosh \varepsilon(p)=\cosh 2 K^{*} \cosh 2 K-\sinh 2 K^{*}$. $\sinh 2 K \cos p, \varepsilon(p) \geqq 0$,

$$
L_{\lambda}^{x}\left((p)_{1, m} \mid(p)_{m+1, n}\right)=(2 \pi)^{-n / 2}(m !(n-m) !)^{-1 / 2} \cdot \operatorname{Pf} B_{\lambda}^{x}\left((p)_{1, m} \mid(p)_{m+1, n}\right),
$$

$P f \equiv$ Pfaffian. $B_{\lambda}^{x}$ is the $(n+1) \times(n+1)$ anti-symmetric matrix with entries

$$
B_{\lambda 1 j}^{x}=\Phi_{-(j-1)}^{\lambda}, \quad j=2, \ldots, n+1, B_{\lambda i+1, j+1}^{x}=A_{i j}^{\lambda} .
$$

$A_{i j}^{\lambda}, 1 \leqq i, j \leqq n$, is the $n \times n$ anti-symmetric matrix with matrix elements

$$
\begin{gathered}
A_{i j}^{\lambda}=m_{-i j}^{\lambda}, 1 \leqq i<j \leqq m, A_{i j}^{\lambda}=-m_{-i j}^{\lambda}, m+1 \leqq i<j \leqq n, \\
A_{i j}^{\lambda}=m_{+i j}^{\lambda}, 1 \leqq i \leqq m, m+1 \leqq j \leqq n, \\
m_{ \pm i j}^{\lambda} \equiv m_{ \pm}^{\lambda}\left(e^{i p_{\imath} \lambda}, e^{i p_{j} \lambda}\right) \chi_{\lambda}\left(p_{i}, p_{j}\right), \\
\chi_{\lambda}\left(k_{1}, \ldots, k_{n}\right)=\chi_{\lambda}\left(k_{1}\right) \ldots \chi_{\lambda}\left(k_{n}\right),
\end{gathered}
$$

$\chi_{\lambda}(k)$ the characteristic function of $[-\pi / \lambda, \pi / \lambda]$ and the function

$$
m_{ \pm}^{\lambda}\left(z_{1}, z_{2}\right)=\frac{\lambda z_{1} z_{2}}{z_{1} z_{2}-1}\left[\Phi_{-}^{\lambda}\left(z_{1}\right) \Phi_{+}^{\lambda}\left(z_{2}\right) \pm \Phi_{-}^{\lambda}\left(z_{2}\right) \Phi_{+}^{\lambda}\left(z_{1}\right)\right]
$$

where $\Phi_{ \pm}^{\lambda}\left(e^{i \lambda p}\right)=\lambda^{\mp 1 / 2} \Phi_{ \pm}\left(e^{i \lambda p}\right), \Phi_{ \pm}(z)=\left[\left(x_{1}-z^{ \pm 1}\right)\left(x_{2}-z^{ \pm 1}\right)\right]^{ \pm 1 / 2}$ with $x_{1}=\operatorname{ctnh} K^{*} \operatorname{ctnh} K, x_{2}=\operatorname{ctnh} K / \operatorname{ctnh} K^{*}$.

For $\lambda=0, S_{2 N}^{L}$ is defined to be the series of Theorem II of [5] which is the same as (2.2) using the $\lambda \rightarrow 0$ limit functions of Appendix B. The above integrals over the distributional kernels of $T_{i j}^{\lambda}$ are symbolic: the product of the singular factors $-i \lambda\left(1-e^{i \lambda\left(p_{i}-p_{j}\right)}\right)^{-1} \chi_{\lambda}\left(p_{i}\right) \chi_{\lambda}\left(p_{j}\right)$ of $m_{+i j}^{\lambda}$ being defined as the tensor product of $H_{\lambda}$ 's, the scaled Hilbert transforms of Appendix C. The above holds for $T>T_{c}$, and a similar representation holds for $T<T_{c}$ (see [5]).

Concerning the convergence and $\lambda \rightarrow 0$ limit (scaling limit) of (2.2) we have

Theorem II.1. a) The series for $S_{k \lambda}$ converges absolutely and uniformly for $\lambda \in[0,1]$ 
and all $S_{i}$ bounded away from zero. b) $S_{k}^{L}$ is the scaling limit of $S_{k \lambda}$, i.e. $\lim _{\lambda \rightarrow 0} S_{k \lambda}=S_{k}^{L}$; the limit can be taken term by term, uniformly for all $s_{i}$ bounded away from zero.

From Theorem II.1 follows

Theorem II.2. $S_{k}^{L}$ manifestly satisfies $\mathrm{O}-\mathrm{S}$ positivity, is invariant under Euclidean translations and has $\mathrm{O}-\mathrm{S}$ symmetry.

The proof of Theorem II.1 will be given in a series of lemmas.

Lemma II.2. Let $c=1+c_{1}+c_{3}, c_{1}$ and $c_{3}$ given by Lemmas $B .2$ and B.3 respectively and let $\rho(s)=\left(1+c_{1}+c_{2}\right)^{2}, c_{2}$ given by Lemma B.2 Then

a) $\left\|T_{m_{J} m_{l}}^{\lambda}\right\| \leqq c(c / \pi)^{\left(m_{i}+m_{J}\right) / 2} \rho\left(s_{j}\right)^{m_{j} / 2} \rho\left(s_{i}\right)^{m_{i} / 2}\left(m_{i}+1\right)^{\left(m_{i}+1\right) / 4}\left(m_{j}+1\right)^{\left(m_{J}+1\right) / 4}$.

$\left(m_{i} ! m_{j} !\right)^{-1 / 2} \sum_{k=0}^{m_{l j}}\left(m_{i}+1\right)^{3 k / 4}\left(m_{j}+1\right)^{3 k / 4} / k !$ where $m_{i j}=\min \left\{m_{i}, m_{j}\right\}$,

b) $\left\|T_{m_{1}}^{\lambda}\right\| \leqq c(c / \pi)^{m_{1} / 2} \rho\left(s_{i}\right)^{m_{1} / 2}\left(m_{i}+1\right)^{\left(m_{1}+1\right) / 4}\left(m_{i} !\right)^{-1 / 2}$,

c) $\left|\theta_{m_{1}}^{\lambda}\right|_{L^{2}} \leqq\left(1 / c_{0} s_{1}\right)^{m_{1} / 2}(2 \pi)^{-1 / 2 m_{1}}\left(m_{1} !\right)^{-1 / 2}\left(m_{1}+1\right)^{\left(m_{1}+1\right) / 4} c^{\left(m_{1}+1\right) / 2}$

with $c_{0}$ given in Lemma B.1.

The proof of Lemma II.2 is given in Appendix A.

$$
\begin{aligned}
\text { Let } m_{i j} & =\min \left(m_{i}, m_{j}\right), \\
F\left(m_{i}, m_{j}\right) & =\sum_{k=0}^{m_{\mathrm{i} j}}(1 / k !)\left[\left(m_{i}+1\right)\left(m_{j}+1\right)\right]^{3 k / 4}
\end{aligned}
$$

and $G(m, \xi)=\xi^{m}(m+1)^{(m+1) / 2} / m !$.

Lemma II.3. Let $\xi_{l}=\frac{c}{\pi} \rho\left(s_{l}\right)+\frac{c}{c_{0} s_{1} 2 \pi}$, then

$$
\left|S_{2 N \lambda}\right| \leqq c^{2 N} \sum_{\left\{m_{l}\right\}_{1}^{N}}\left[\prod_{l=1}^{2 N-1} G\left(m_{l}, \xi_{l}\right)\right]\left[\prod_{l=1}^{2 N-1} F\left(m_{l}, m_{l+1}\right)\right] \text {. }
$$

Proof. Follows from Lemmas II.1 and II.2.

To study the convergence of the series in Eq. (2.3) define

$$
H\left(m_{2}, \xi\right)=\sum_{m_{1}=0}^{\infty} F\left(m_{1}, m_{2}\right) G\left(m_{1}, \xi\right) .
$$

Lemma II.4. $H\left(m_{2}, \xi\right)<\eta(\xi) e^{v(\xi) m_{2}}$, where

$$
\eta(\xi)=\gamma_{1} \exp \left(\gamma_{2} \xi^{2}+\gamma_{3} \xi^{4 / 3}\right) \text { and } v(\xi)=\gamma_{3} \xi^{4 / 3}
$$

for some numerical constants $\gamma_{1}, \gamma_{2}, \gamma_{3}$.

Proof. Using the identity $\sum_{m_{1}=0}^{\infty} \sum_{k=0}^{m_{1} 2}=\sum_{k=0}^{m_{2}} \sum_{m_{1}=k}^{\infty}$ we have

$$
H\left(m_{2}, \xi\right)=\sum_{k=0}^{m_{2}} \frac{1}{k !}\left(m_{2}+1\right)^{3 k / 4} I(k, \xi), I(k, \xi)=\sum_{m_{1}=k}^{\infty} \frac{1}{m_{1} !}\left(m_{1}+1\right)^{\left(m_{1}+1+(3 / 2) k\right) / 2} \text {. }
$$


Using the inequality

$$
\begin{aligned}
& n^{n} e^{-(n-1)} \leqq n ! \leqq(n+1)^{(n+1)} e^{-n}, I(k, \xi) \leqq \sum_{m=k}^{\infty}(\xi e)^{m} /(m+1)^{(m-1-(3 / 2) k) / 2} \\
& =(\xi e)^{k} \sum_{m=0}^{\infty}(\xi e)^{m}(m+k+1)^{(1+k / 2)} /(m+k+1)^{m / 2} \\
& \leqq(\xi e)^{k} \sum_{m=0}^{\infty}(\xi e)^{m} e^{(m+k) / 2} k^{k / 4} e^{(m+1) / 4} /(m !)^{1 / 2} \\
& =e^{1 / 4}\left(\xi e^{3 / 2}\right)^{k} k^{k / 4} \sum_{m=0}^{\infty}\left(\xi e^{7 / 4}\right)^{m} /(m !)^{1 / 2} .
\end{aligned}
$$

From this we conclude, using Schwarz's inequality, that there are numerical constants $\alpha_{1}, \alpha_{2}, \alpha_{3}$ such that $I(k, \xi) \leqq \alpha_{1}\left(\alpha_{2} \xi\right)^{k} k^{k / 2} \exp \left(\alpha_{3} \xi^{2}\right)$. Therefore,

$$
\begin{aligned}
H\left(m_{2}, \xi\right) & \leqq \alpha_{1} e^{\alpha_{3} \xi^{2}} \sum_{k=0}^{\infty} \frac{1}{k !}\left(m_{2}+1\right)^{3 k / 4}\left(\alpha_{2} \xi\right)^{k} k^{k / 4} \\
& \leqq \alpha_{1} e^{\alpha_{3} \xi^{2}} \sum_{k=0}^{\infty} \frac{1}{(k !)^{3 / 4}}\left(m_{2}+1\right)^{3 k / 4}\left(\alpha_{2} \xi\right)^{k} e^{(k-1) / 4} .
\end{aligned}
$$

by (2.4). Thus, by Hölder's inequality, there are constants such that

$$
H\left(m_{2}, \xi\right) \leqq \alpha_{4} e^{\alpha_{3} \xi^{2}} e^{\alpha_{5} \xi^{4 / 3}\left(m_{2}+1\right)} .
$$

Finally the convergence of the series of Lemma II.3 is established in Lemma II.5 below. Define recursively $\xi_{12 \ldots j}$ by $\xi_{12 \ldots j}=\xi_{j} \exp \left|v\left(\xi_{12, \ldots j-1}\right)\right|$.

Lemma II.5. $\left|S_{2 N \lambda}\right| \leqq \prod_{j=1}^{2 N-1} \eta\left(\xi_{12 \ldots j}\right)$.

Proof. Summing over $m_{1}$ in (2.3) and using Lemma II.4 we have

$$
\begin{aligned}
&\left|S_{2 N \lambda}\right| \leqq \eta\left(\xi_{1}\right) \sum_{\left\{m_{l}\right\}_{3}^{N-1}}\left[\prod_{l=3}^{2 N-1} G\left(m_{l}, \xi_{l}\right)\right]\left[\prod_{l=3}^{2 N-1} F\left(m_{l}, m_{l+1}\right)\right] \\
& \cdot \sum_{m_{2}=0}^{\infty} F\left(m_{2}, m_{3}\right) G\left(m_{2}, \xi_{12}\right) .
\end{aligned}
$$

In obtaining (2.5) we have used the fact that $G\left(m_{2}, \xi_{2}\right) e^{v\left(\xi_{1}\right) m_{2}}=G\left(m_{2}, \xi_{12}\right)$. By Lemma II.4 the sum over $m_{2}$ is bounded by $\eta\left(\xi_{12}\right) e^{v\left(\xi_{12}\right) m_{3}}$. By repeated use of this process we arrive at

$$
\left|S_{2 N \lambda}\right| \leqq \eta\left(\xi_{1}\right) \eta\left(\xi_{12}\right) \ldots \eta\left(\xi_{12 \ldots 2 N-2}\right) \sum_{m_{2 N-1=0}}^{\infty} G\left(m_{2 N-1}, \xi_{12 \ldots 2 N-1}\right)
$$

and from Lemma II.4 with $m_{2}=0$

$$
\sum_{m_{2 N-1}=0}^{\infty} G\left(m_{2 N-1}, \xi_{12 \ldots 2 N-1}\right) \leqq \eta\left(\xi_{12 \ldots 2 N-1}\right) .
$$

Lemma II.5 completes the proof of Theorem II.1a. We now turn to the proof of Theorem II.1b. By the uniform convergence in $\lambda$ of the series for $S_{2 N \lambda}$ (established in a) it is sufficient to show convergence for $\lambda \rightarrow 0$ of a general term of $S_{2 N \lambda}$ of 
Lemma II.1 which follows immediately from the following lemma:

Lemma II.6. Let $\rightarrow$ denote convergence in the strong $L^{2}$ operator topology. Then as $\lambda \rightarrow 0$.
a) $T_{i j}^{\lambda} \rightarrow T_{i j}$,
b) $T_{i}^{\lambda} \rightarrow T_{i}$,
c) $\theta_{i}^{\lambda} \rightarrow \theta_{l}$ in $L^{2}$

uniformly for all imaginary time difference variables bounded away from zero. The proof of Lemma II.6 is given in Appendix A.

\section{O-S Temperedness of $S_{k}^{L}$ and Clustering}

The following lemma on the factorization of scaling limit Fermion matrix elements of the spin operator is used to obtain upper bounds on $S_{2}^{L}$ sufficient to guarantee $\mathrm{O}-\mathrm{S}$ temperedness.

Lemma III.1. Let $D$ be the $n+1$ dimensional anti-symmetric square matrix with matrix elements $\Delta_{-}\left(p_{i}, p_{j}\right)=\left(\omega\left(p_{i}\right)-\omega\left(p_{j}\right)\right) /\left(p_{i}+p_{j}\right), 0 \leqq i<j \leqq n, n$ odd. Then Pfaffian $D \equiv \operatorname{Pf} D=\prod_{0 \leqq i<j \leqq n} \Delta_{-}\left(p_{i}, p_{j}\right)$.

Remark. By taking $p_{0} \rightarrow \infty$ we obtain the Pfaffian appropriate for $T^{+}$with 1 's in the first line after taking out an overall factor of

$$
\prod_{i=0}^{n}\left(p_{i}-i m\right)^{-1 / 2} \text { from } \quad \text { Pf } B_{0}^{x}
$$

Proof. Introduce the rapidity variable $\theta$ by $p=m \sinh 2 \theta$, then $\Delta_{-}\left(p_{i}, p_{j}\right)=$ $\tanh \left(\theta_{i}-\theta_{j}\right)$. By definition the Pfaffian is then

$$
D=\sum_{p \in P}(-1)^{\sigma_{p}} \prod_{k=0}^{(n-1) / 2} \tanh \left(\theta_{p_{1 k}}-\theta_{p_{2 k}}\right),
$$

where $P$ is a partition of $\{0,1, \ldots, n\}$ into two disjoint classes $\left\{p_{10}, p_{11}, \ldots, \mathrm{p}_{1(n-1) / 2}\right\}$, $\left\{p_{20}, p_{21}, \ldots, p_{2(n-1) / 2}\right\}$ such that $p_{1 k}<p_{2 k}$ and $(-1)^{\sigma_{p}}$ is the sign of the permutation $\{0,1, \ldots, n\} \rightarrow\left\{p_{10}, p_{20}, \ldots, p_{11}, p_{21}, \ldots, p_{1(n-1) / 2}, p_{2(n-1) / 2}\right\}$.

Let $f_{i}=\tanh \theta_{i}$, then

$$
D=\sum_{p \in P}(-1)^{\sigma_{p}} \prod_{k=0}^{(n-1) / 2}\left(f_{p_{1 k}}-f_{p_{2 k}}\right) /\left(1-f_{p_{1 k}} f_{p_{2 k}}\right) .
$$

Multiply $D$ by the symmetric $\prod_{0 \leqq i<j \leqq n}\left(1-f_{i} f_{j}\right)$ to obtain

$$
\begin{array}{r}
B \equiv D \prod_{0 \leqq i<j \leqq n}\left(1-f_{i} f_{j}\right)=\sum_{p \in P}(-1)^{\sigma_{p}} \prod_{k=0}^{(n-1) / 2}\left(f_{p_{1 k}}-f_{p_{2 k}}\right) \prod_{0 \leqq i<j \leqq n}\left(f_{p_{i}}-f_{p_{j}}\right) \\
(i, j) \neq\left(p_{1 k}, p_{2 k}\right) .
\end{array}
$$

For fixed $l$ the degree of $f_{l}$ is one in the first factor and $n-1$ in the second factor. $B$ is an anti-symmetric polynomial of degree $n$ in each variable $f_{0}, f_{1}, \ldots, f_{n}$ and $B=0$ if $f_{i}=f_{j}$ for some $i$. Thus $B$ has the form $B=c \prod_{0 \leqq i<j<n}\left(f_{i}-f_{j}\right)$, where $c$ is 
a constant so that $D=c \prod_{0 \leqq i<j<n} \tanh \left(\theta_{i}-\theta_{j}\right)$. Taking successively the limits $\theta_{0} \rightarrow \infty, \theta_{1} \rightarrow \infty, \ldots, \theta_{n} \rightarrow \infty$ we obtain $c=1$.

Concerning $S_{k}^{L}$ we have

Theorem III.1. The $S_{k}^{L}$ satisfy $\mathrm{O}-\mathrm{S}$ temperedness. In particular, let $S_{2}^{L}(s) \equiv$ $S_{2}^{L}(0, s), s>0$, denote the difference variable 2-point function and $S_{2}^{f}(s) \equiv$ $\frac{1}{2 \pi} \int_{-\infty}^{\infty} e^{-s \omega(p)} \frac{d p}{\omega(p)}$, the free particle 2-point Schwinger function for mass $m$. Then

$$
\begin{gathered}
0<S_{2}^{L}-1<\sum_{\substack{n=2 \\
n \text { even }}}^{\infty} \frac{\left(S_{2}^{f}\right)^{n}}{n !}<e^{S_{2}^{f}}-S_{2}^{f}-1 \text { for } T^{-}, \\
S_{2}^{f}<S_{2}^{L}<\sum_{n \text { odd }}^{\infty} \frac{\left(S_{2}^{f}\right)^{n}}{n !}<e^{S_{2}^{f}}-1 \text { for } T^{+}
\end{gathered}
$$

and for $T^{+}$and $T^{-}, s \in(0,1], S_{2}^{L}(s)<c / s^{1 / \pi}$ for some constant $c$.

Proof. As $\left.\mid \Delta_{-}(p, q)\right\} \leqq 1$ for all $p, q \in R$, the upper bound on $S_{2}^{L}$ follows by using Lemma III.1 to bound the Pfaffians $\operatorname{Pf} B_{0}^{x}\left(\phi \mid(p)_{1, m_{1}}\right)$ occurring in the series representation (2.2). By rotational invariance (see Theorem IV.1) the bounds hold in the $s, x$ plane where $s$ is now to be interpreted as the Euclidean distance.

From the existence of the $\lambda \rightarrow 0$ limit (Theorem II.1b), and using the upper bounds on $S_{2}^{L}$ in the inequalities of [6], it follows that $S_{k}^{L}$ satisfies the O-S temperedness axiom $E-0^{\prime}$ [7]. In order that the inequalities of [6] apply, the $\lambda \rightarrow 0$ limit is taken, with $s_{i}, x_{i}$ rational, through a sequence $\left\{\lambda_{j}\right\}$ such that $s_{i} / \lambda_{j}, x_{i} / \lambda_{j}, 1 \leqq i \leqq k-1$ are integers; by continuity the bounds on $S_{k}^{L}$ hold for all $s_{i}, x_{i}, s_{i}>0$.

We now give a cluster decomposition property in the space-imaginary time variables for $T^{+}$which implies the uniqueness of the vacuum of the reconstructed Wightman theory (see [10]).

Theorem III.2. For $T^{+}$, let $f \in C_{0}^{\infty}\left(R_{+}^{2 n}\right), g \in C_{0}^{\infty}\left(R_{+}^{2 m}\right)$ with the supports strictly contained in $R_{+}^{2 n}, R_{+}^{2 m}$ where

$$
R_{+}^{2 l}=\left\{\left(x_{1}^{\prime}, s_{1}^{\prime} ; \ldots ; x_{l}^{\prime}, s_{l}^{\prime}\right) \in R^{2 l}, 0<s_{1}^{\prime}<s_{2}^{\prime}<\ldots<s_{l}^{\prime}\right\}, R_{+}^{0} \equiv C .
$$

Then

$$
\lim _{t \rightarrow \infty} S_{m+n}^{L}\left(\theta^{\sim} f \otimes T_{t} g\right)=S_{n}^{L}\left(\theta^{\sim} f\right) S_{m}(g)
$$

where

$$
\theta^{\sim} f\left(x_{1}^{\prime}, s_{1}^{\prime} ; \ldots ; x_{n}^{\prime}, s_{n}^{\prime}\right)=\overline{f\left(x_{n}^{\prime},-s_{n}^{\prime} ; \ldots ; x_{1}^{\prime},-s_{1}^{\prime}\right)}
$$

and

$$
T_{t} g\left(x_{1}^{\prime}, s_{1}^{\prime} ; \ldots ; x_{m}^{\prime}, s_{m}^{\prime}\right)=g\left(x_{1}^{\prime}, s_{1}^{\prime}-t ; \ldots ; x_{m}^{\prime}, s_{m}^{\prime}-t\right), \quad t>0 .
$$

Proof. $S_{k}^{L}$ is approximated by $S_{k}^{\lambda}$ uniformly for all $s_{i}^{\prime}-s_{i-1}^{\prime}$ bounded away from 
zero (by Lemma II.6) and $S_{k \lambda}$ satisfies pointwise clustering; thus $S_{k}^{L}$ satisfies pointwise clustering and as $S_{k}^{L}$ is uniformly bounded for all $s_{i+1}^{\prime}-s_{i}^{\prime}$ bounded away from zero (3.1) follows from the Lebesgue bounded convergence theorem.

\section{Rotational Invariance of $S_{k}^{L}$}

Writing the series representation for $S_{k}^{L}$ in terms of the rapidity variables $p=m \sinh 2 \theta, \omega(p)=m \cosh 2 \theta$, we give a simple proof of rotational invariance for $k=2$. For $k>2$ the same idea is used but the singular Hilbert transform $H_{0}$ in rapidity variables is regularized using the operator $H_{\varepsilon}^{R}$ of Appendix $\mathrm{C}$ and we give the proof in Appendix D.

Theorem IV.1. Let $S_{k}^{L}\left(x_{1}, s_{1} ; \ldots ; x_{k-1}, s_{k-1}\right)$ denote the Schwinger function in the difference variables. Then

$$
\sum_{i=1}^{k-1}\left(x_{i} \partial S_{k}^{L} / \partial s_{i}-s_{i} \partial S_{k}^{L} / \partial x_{i}\right)=0
$$

and the derivatives can be calculated term by term in (2.2).

Proof. 2-point function. Let $\boldsymbol{\theta}_{n}=\left(\theta_{1}, \ldots, \theta_{n}\right)$ denote the rapidity variables. Then $S_{2}^{L}$ can be written

$$
S_{2}^{L}(x, s)=\sum_{n}^{\infty} S_{2}^{L(n)} \equiv \sum_{n}^{\infty} \int I_{n} e^{K_{n}} d \theta_{n}
$$

where $I_{n} \equiv I_{n}\left(\theta_{n}\right)$ is a function of the difference variables only, since $\left(\omega\left(p_{i}\right)-\omega\left(p_{j}\right)\right)$ / $\left(p_{i}+p_{j}\right)=\tanh \left(\theta_{i}-\theta_{j}\right)$ and $d p / \omega=2 d \theta$, and

$$
K_{n} \equiv K_{n}\left(s, x, \boldsymbol{\theta}_{n}\right)=-m s \sum_{i=1}^{n} \cosh 2 \theta_{i}+i m x \sum_{i=1}^{n} \sinh 2 \theta_{i}, s>0 .
$$

With $\mathbf{n}=(1,1, \ldots, 1)$ and $\nabla_{\theta}=\left(\partial / \partial \theta_{1}, \ldots, \partial / \partial \theta_{n}\right)$, upon differentiating inside the infinite sum and integral and integrating by parts, we have

$$
x \frac{\partial S_{2}^{L}}{\partial s}-s \frac{\partial S_{2}^{L}}{\partial x}=\frac{i}{2} \sum_{n}^{\infty} \int I_{n}\left(\mathbf{n} \cdot \nabla_{\theta} e^{K_{n}}\right) d \boldsymbol{\theta}_{n}=-\frac{i}{2} \sum_{n}^{\infty} \int\left(\mathbf{n} \cdot \nabla_{\theta} I_{n}\right) e^{K_{n}} d \boldsymbol{\theta}_{n}=0 .
$$

To justify the term by term differentiation and the interchange of derivative and integral, consider for example $\partial S_{2} / \partial s$. For $b>0$, using Taylor's theorem for $\exp \left(-s \sum_{i=1}^{n} \omega_{i}\right), \omega_{i} \equiv \omega\left(p_{i}\right)$, we can write

$$
\begin{aligned}
& b^{-1}\left(S_{2}^{L}(s+b)-S_{2}^{L}(s)\right)-\sum_{n}^{\infty} \int I_{n}\left(-\sum_{i=1}^{n} \omega_{i}\right) \exp \left(-s \sum_{i=1}^{n} \omega_{i}+i x \sum_{i=1}^{n} p_{i}\right) d^{n} p \\
& =b^{-1} \sum_{n}^{\infty} \int I_{n} \exp \left(i x \sum_{i=1}^{n} p_{i}\right)\left[\int_{s}^{s+b}(s+b-t)\left(-\sum_{i=1}^{n} \omega_{i}\right)^{2}\right. \\
& \left.\quad \cdot \exp \left(-t \sum_{i=1}^{n} \omega_{i}\right) d t\right] d^{n} p .
\end{aligned}
$$


For $0<\delta<s, u e^{-s u}$ and $u^{2} e^{-s u}$ are bounded by $M_{\delta} e^{-(s-\delta) u}$ for some $0<M_{\delta}<\infty$ so that the series on the left of (4.1) is absolutely convergent since $M_{\delta} \sum_{n}^{\infty} \int\left|I_{n}\right| \exp \left(-(s-\delta) \sum_{i=1}^{n} \omega_{i}\right) d^{n} p$ is (by Theorem II.1a); the series on the right side is bounded by $(b / 2) M_{\delta} \sum_{n}^{\infty} \int\left|I_{n}\right| \exp \left[-(s-\delta) \sum_{i=1}^{n} \omega_{i}\right] d^{n} p$ and is absolutely convergent (again by Theorem II.1a) so that the $b \downarrow 0$ limit of (4.1) exists and is zero. A similar analysis holds for $b<0$ and $\partial / \partial x$.

\section{Appendix A}

In this appendix we prove Lemmas II.2 and II.6. The key to the proof of Lemma II.2 uses the combinatorial Lemma A.1 below which is an expansion of $B_{\lambda}^{x}$ in the number of singular functions. In bounding $T_{j i}^{\lambda}$ the singular functions are bounded by using the norm non-increasing property of the scaled Hilbert transforms of Appendix $\mathrm{C}$ and the non-singular Pfaffians are majorized by Hadamard's inequality. In this appendix we abbreviate $m_{ \pm}^{\lambda}\left(e^{i p_{i}}, e^{i p_{j}}\right)$ by $m_{ \pm}^{\lambda}\left(p_{i}, p_{j}\right)$ and let $\Delta_{i_{1} \ldots i}(p)_{1, n}$ denote the set $\left\{p_{1}, p_{2}, \ldots, p_{n}\right\}$ with $p_{i_{1}}, \ldots, p_{i_{1}}$ deleted. We have

Lemma A.1. Let $n_{2}>0$ be even and $n_{1}$ odd. Then

$$
\begin{aligned}
& \operatorname{Pf} B_{\lambda}^{x}\left(\left(-p^{2}\right)_{1, n_{2}} \mid\left(p^{1}\right)_{1, n_{1}}\right)=\sum_{m=0}^{n_{12}} \sum_{\left(i_{1} j_{1}\right) \ldots\left(i_{m} j_{m}\right)}^{\prime}(-1)^{\sigma\left(i_{1} j_{1}\right) \ldots\left(i_{m} j_{m}\right)} \\
& \cdot \operatorname{Pf} B\left(\Delta_{i_{1} \ldots i_{m}}\left(-p^{2}\right)_{1, n_{2}}\right) \operatorname{Pf} B\left(\Delta_{j_{1} \ldots j_{m}}\left(p^{\prime}\right)_{1, n_{1}}\right) \prod_{k=1}^{m} m_{+}^{\lambda}\left(-p_{i_{k}}^{2}, p_{j_{k}}^{1}\right),
\end{aligned}
$$

where, with $q^{1}=p^{1}, q^{2}=-p^{2}$,

$B\left(\Delta_{k_{1} \ldots k_{m}}\left(q^{l}\right)_{1, n_{l}}\right)=\left\{\begin{array}{l}B_{\lambda}\left(\Delta_{k_{1} \ldots k_{m}}\left(q^{l}\right)_{1, n_{l}}\right), \text { if } m \text { is even and or } m \text { is odd and } l=1 \\ B_{\lambda}^{x}\left(\phi \mid \Delta_{k_{1} \ldots k_{m}}\left(q^{l}\right)_{1, n_{l}}\right), \text { if } m \text { is even and } l=1 \text { or } m \text { is odd and } l=2\end{array}\right\}$ and $B_{\lambda}\left((p)_{1, n}\right)$ (n even) is the $n \times n$ anti-symmetric matrix with elements $m_{-}^{\lambda}\left(p_{i}, p_{j}\right)$ for $1 \leqq i<j \leqq n$. The second summation in $(A . I)$ is over all possible configurations of $m$ pairs $(i j)$ with $1 \leqq i \leqq n_{2}$ and $1 \leqq j \leqq n_{1} ;(-1)^{\sigma\left(i_{1} j_{1}\right) \ldots\left(i_{m} j_{m}\right)}$ is the sign of the permutation bringing together the pairs $\left(i_{i} \tilde{\tilde{j}}_{1}\right), \ldots,\left(i_{k} \tilde{j}_{k}\right)$ starting from the arrangement $\left\{1,2, \ldots, n_{2}, \tilde{1}, \tilde{2}, \ldots, \tilde{n}_{1}\right\}$ and $n_{12}=\min \left\{n_{1}, n_{2}\right\}$.

Proof. Group the points $\left(-p^{2}\right)_{1, n_{2}}$ into a set $A$ and $\left(p^{1}\right)_{1, n_{1}}$ in $B$. Then, $P f B_{\lambda}^{x}$ $\left(\left(-p^{2}\right)_{1, n_{2}} \mid\left(p^{1}\right)_{1, n_{1}}\right)$ can be pictured as a sum of graphs involving contractions of points in $A, B$ and a point outside $A$ and $B$, call it 0 . The contraction function within $A$ or $B$ is $m_{-}^{\lambda}$. The contraction between a point in $A$ and one in $B$ is $m_{+}^{\lambda}$, and the contraction between 0 and a point in $A$ or $B$ is $\Phi_{-}^{\lambda}$. The proof of the lemma follows by resummation of all graphs with $0,1,2, \ldots, n_{12}$ contractions between the points of $A$ and $B$.

Proof of Lemma II.2. a) Expanding $P f B_{\lambda}^{x}$ of $T_{j i}^{\lambda}$ according to Lemma A.1 we have 


$$
\begin{gathered}
\operatorname{Pf} B_{\lambda}^{x}\left(\left(-p^{j}\right)_{1, m_{j}} \mid\left(p^{i}\right)_{1, m_{i}}\right)=\sum_{k=0}^{m_{i j}} \sum_{\left(\alpha_{1} \beta_{1}\right) \ldots\left(\alpha_{k} \beta_{k}\right)}(-1)^{\sigma\left(\alpha_{1} \beta_{1}\right) \ldots\left(\alpha_{k} \beta_{k}\right)} \\
\cdot \prod_{r=1}^{k} m_{+}^{\lambda}\left(-p_{\alpha_{r}}^{j}, p_{\beta_{r}}^{i}\right) . \\
\left\{\begin{array}{l}
P f B_{\lambda}\left(\Delta_{\alpha_{1} \ldots \alpha_{k}}\left(-p^{j}\right)_{1, m_{j}}\right) P f B_{\lambda}^{x}\left(\phi \mid \Delta_{\beta_{1} \ldots \beta_{k}}\left(p^{i}\right)_{1, m_{i}}\right) \text { if } k \text { is even } \\
P f B_{\lambda}^{x}\left(\phi \mid \Delta_{\alpha_{1} \ldots \alpha_{k}}\left(-p^{j}\right)_{1, m_{j}}\right) P f B_{\lambda}\left(\Delta_{\beta_{1} \ldots \beta_{k}}\left(p^{i}\right)_{1, m_{i}}\right) \text { if } k \text { is odd }
\end{array}\right\} .
\end{gathered}
$$

The $k=0$ term above is $\operatorname{Pf} B_{\lambda}\left(\left(-p^{j}\right)_{1, m_{\mathrm{J}}}\right) \operatorname{Pf} B_{\lambda}^{x}\left(\phi \mid\left(p^{i}\right)_{1, m_{\mathrm{r}}}\right)$ and the $k=m_{i j}$ term is

$$
\begin{gathered}
P f B_{\lambda}^{x}\left(\phi \mid \Delta_{\beta_{1} \ldots \beta m_{j}}\left(p^{i}\right)_{1, m_{j}}\right) \text { if } m_{j}<m_{i}, \\
\operatorname{Pf} B_{\lambda}^{x}\left(\phi \mid \Delta_{\alpha_{1} \ldots \alpha_{m_{i}}}\left(-p^{j}\right)_{1, m_{j}}\right) \text { if } m_{i}<m_{j} .
\end{gathered}
$$

Let $T_{m_{j} m_{i}}^{\lambda\left(\alpha_{1} \beta_{1}\right) \ldots\left(\alpha_{k} \beta_{k}\right)}\left(x_{j}, s_{j} ; x_{i}, s_{i}\right)$ (abbreviated $\left.T_{j i}^{\lambda(\alpha \beta)_{k}}\right)$ be defined by

$$
\begin{gathered}
\left(T_{j i}^{\lambda(\alpha \beta)_{k}} f\right)\left(\left(p^{j}\right)_{1, m_{j}}\right)=(2 \pi)^{(-1 / 2)\left(m_{i}+m_{j}\right)}\left(m_{i} ! m_{j} !\right)^{-1 / 2} \int \prod_{r=1}^{k} m_{+}^{\lambda}\left(-p_{\alpha_{r}}^{j}, p_{\beta_{r}}^{i}\right) \\
\cdot\left\{\begin{array}{l}
P f B_{\lambda}\left(\Delta_{\alpha_{1} \ldots \alpha_{k}}\left(-p^{j}\right)_{1, m_{j}}\right) P f B_{\lambda}^{x}\left(\Delta_{\beta_{1} \ldots \beta_{k}}\left(p^{i}\right)_{1, m_{i}}\right) \text { if } k \text { is even } \\
\left.P f B_{\lambda}^{x}\left(\phi \mid \Delta_{\alpha_{1} \ldots \alpha_{k}}\left(-p^{j}\right)_{1, m_{j}}\right) P f B_{\lambda}\left(\Delta_{\beta_{1} \ldots \beta_{k}}\left(p^{i}\right)_{1, m_{1}}\right) \text { if } k \text { is odd }\right\}
\end{array}\right. \\
\cdot \exp \left\{-\frac{1}{2} \sum_{k=i, j} \sum_{l=1}^{m_{k}}\left(\omega_{\lambda}\left(p_{l}^{k}\right) s_{k}+i p_{l}^{k} x_{k}\right)\right\} f\left(\left(p^{i}\right)_{1, m_{l}}\right) d^{m_{1}} p^{i}
\end{gathered}
$$

Then

$$
T_{j i}^{\lambda}=\sum_{k=0}^{m_{l j}} \sum_{\left(\alpha_{1} \beta_{1}\right)_{1} \ldots\left(\alpha_{k} \beta_{k}\right)}^{\prime} T_{j i}^{\lambda(\alpha \beta)_{k}}(-1)^{\sigma\left(\alpha_{1} \beta_{1}\right) \ldots\left(\alpha_{k} \beta_{k}\right)} .
$$

Since clearly $\left\|T_{j i}^{\lambda(\alpha \beta)_{k}}\right\|$ is independent of the particular sequence $\left(\alpha_{1} \beta_{1}\right) \ldots\left(\alpha_{k} \beta_{k}\right)$ we have

$$
\left\|T_{j i}^{\lambda}\right\| \leqq \sum_{k=0}^{m_{l j}}\left\|T_{j i}^{\lambda k}\right\| \sum_{\left(\alpha_{1} \beta_{1}\right) \ldots\left(\alpha_{k} \beta_{k}\right)}^{\prime} 1,
$$

where $T_{j i}^{\lambda k}$ is $T_{j i}^{\lambda(\alpha \beta)_{k}}$ for the particular choice $\alpha_{l}=\beta_{l}=l$. Noting that

$$
\begin{aligned}
\sum_{\left(\alpha_{1} \beta_{1}\right) \ldots\left(\alpha_{k} \beta_{k}\right)}^{\prime} 1 & =(1 / k !) m_{i}\left(m_{i}-1\right) \ldots\left(m_{i}-k+1\right) m_{j}\left(m_{j}-1\right) \ldots\left(m_{j}-k+1\right) \\
& \leqq(1 / k !) m_{i}^{k} m_{j}^{k},
\end{aligned}
$$

and substituting from (A.6) below in (A.5) gives

$$
\begin{aligned}
\left\|T_{j i}^{\lambda}\right\| & \leqq c(c / \pi)^{(1 / 2)\left(m_{i}+m_{j}\right)} \rho\left(s_{j}\right)^{m_{j} / 2} \rho\left(s_{i}\right)^{m_{\imath} / 2}\left(m_{i} ! m_{j} !\right)^{-1 / 2} \\
& \cdot \sum_{k=0}^{m_{i j}}(1 / k !)\left(m_{i}-k+1\right)^{(1 / 4)\left(m_{\imath}-k+1\right)}\left(m_{j}-k+1\right)^{(1 / 4)\left(m_{J}-k+1\right)} m_{i}^{k} m_{j}^{k} .
\end{aligned}
$$

The sum is bounded by

$$
\left(m_{i}+1\right)^{(1 / 4)\left(m_{\imath}+1\right)}\left(m_{j}+1\right)^{(1 / 4)\left(m_{J}+1\right)} \sum_{k=0}^{m_{l j}}(k !)^{-1}\left(m_{i}+1\right)^{(3 / 4) k}\left(m_{j}+1\right)^{(3 / 4) k}
$$


Now from Lemma B.2b,c.

$$
\begin{gathered}
\left\|A_{\mathbf{r}^{\prime}}^{\lambda}\left(s_{j}\right)\right\| \leqq \prod_{s=1}^{k}\left\|\Phi_{\mathbf{r}^{\prime} s}^{\lambda} \exp \left\{-\frac{1}{2} \omega_{\lambda} s_{j}\right\}\right\|_{L^{\infty}(R)} \leqq\left(1+c_{1}+c_{2}\right)^{k}, \\
\left\|B_{\mathbf{r}^{\prime}}^{\lambda}\left(s_{i}\right)\right\| \leqq \prod_{t=1}^{k}\left\|\Phi_{\mathbf{r}_{t}}^{\lambda} \exp \left\{-\frac{1}{2} \omega_{\lambda} s_{i}\right\}\right\|_{L^{\infty}(R)} \leqq\left(1+c_{1}+c_{2}\right)^{k},
\end{gathered}
$$

and letting $\rho(s)^{1 / 2}=1+c_{1}+c_{2}$ we have $\left\|R_{j i}^{\lambda k}\right\| \leqq 2^{k} \rho\left(s_{j}\right)^{k / 2} \rho\left(s_{i}\right)^{k / 2}$.

Using Lemma B.3 and Hadamard's inequality to bound $W_{j}^{\lambda k}$ and Lemmas B.2b, B.3 and Hadamard's inequality with $c_{4}=c_{1}+c_{3}$ to bound $V_{i}^{\lambda k}$ we have, for $k$ even,

$$
\begin{array}{r}
\left\|T_{j i}^{\lambda k}\right\| \leqq\left\|W_{j}^{\lambda k}\right\|\left\|R_{j i}^{\lambda k}\right\|\left\|V_{i}^{\lambda k}\right\| \leqq(2 \pi)^{-m_{J} / 2}\left(m_{j} !\right)^{-1 / 2} c_{3}^{(1 / 2)\left(m_{J}-k\right)}\left(m_{j}-k\right)^{\left(m_{J}-k\right) / 4} \\
\cdot 2^{k} \rho\left(s_{j}\right)^{k / 2} \rho\left(s_{i}\right)^{k / 2} \cdot(2 \pi)^{-m_{i} / 2}\left(m_{i} !\right)^{-1 / 2} c_{4}^{\left(m_{i}-k+1\right) / 2}\left(m_{i}-k+1\right)^{\left(m_{i}-k+1\right) / 4}
\end{array}
$$

For $k$ odd the factor $\left(m_{j}-k\right)^{1 / 4\left(m_{j}-k\right)}\left(m_{i}-k+1\right)^{1 / 4\left(m_{i}-k+1\right)}$ is to be replaced by $\left(m_{j}+1-k\right)^{1 / 4\left(m_{j}+1-k\right)}\left(m_{i}-k\right)^{1 / 4\left(m_{i}-k\right)}$. In both cases the factor is majorized by $\left(m_{i}-k+1\right)^{1 / 4\left(m_{i}-k+1\right)}\left(m_{j}-k+1\right)^{1 / 4\left(m_{j}-k+1\right)}$, and upon letting $c=1+c_{1}+c_{3}$, we have

$$
\begin{aligned}
\left\|T_{j l}^{\lambda k}\right\| \leqq & c(c / \pi)^{(1 / 2)\left(m_{i}+m_{j}\right)} \rho\left(s_{j}\right)^{m_{j} / 2} \rho\left(s_{i}\right)^{m_{t} / 2}\left(m_{i} ! m_{j} !\right)^{-1 / 2} \\
& \cdot\left(m_{i}-k+1\right)^{(1 / 4)\left(m_{l}-k+1\right)}\left(m_{j}-k+1\right)^{(1 / 4)\left(m_{j}-k+1\right)} .
\end{aligned}
$$

Proof of Lemma II.6: a) By the expansion of $T_{j i}^{\lambda}$ of (A.4) it is sufficient to show the strong operator convergence of the general term $T_{j i}^{\lambda k}$ of Lemma A.2. By the norm boundedness in $\lambda \in[0,1]$ of the factors in the decompositions $T_{j i}^{\lambda k}=W_{j i}^{\lambda k} R_{j i}^{\lambda k}$ $V_{i}^{\lambda k}$ and $R_{j i}^{\lambda k}=\sum_{\mathbf{r}} A_{\mathbf{r}^{\prime}}^{\lambda} H_{\lambda}^{k} B_{\mathbf{r}}^{\lambda}$ (see (A.7)) strong convergence of $T_{j i}^{\lambda k}$ follows from the strong convergence of the factors $W_{j}^{\lambda}, V_{i}^{\lambda} A_{\mathbf{r}^{\prime}}^{\lambda}, H_{\lambda}^{k}$ and $B_{\mathbf{r}}^{\lambda}$. The multiplication operators converge strongly by the pointwise convergence of Lemma B.4. Note that the convergence is uniform in the $s_{i}$ variables bounded away from zero. $H_{\lambda}^{k}=\prod_{i=1}^{k} H_{\lambda i}$, where $H_{\lambda i}$ is the scaled Hilbert transform $H_{\lambda}$ of Lemma C.1 acting on the $i$-th variable. Since $\left\|H_{\lambda}^{k}\right\| \leqq 1$ it is enough to show pointwise convergence for a dense set $D$ which we take as finite linear combinations of product functions. The strong operator convergence of $H_{\lambda}$ on $L^{2}(R)$ given by Lemma C.1b implies the pointwise convergence of $H_{\lambda}^{k}$ on $D$ which in turn implies the strong operator convergence of $H_{\lambda}^{k}$.

b) follows from the pointwise convergence of the integrand of $T_{i} f$ using Lemma B.4 and the Lebesgue bounded convergence theorem using B.2b, B.3 and Hadamard's inequality and Lemma B.1.

c) follows from Lemma B.4 and using B.2b, B.3 and Hadamard's inequality and Lemma B.1.

\section{Appendix B}

In this appendix we establish bounds and limits of various scaling functions used in Sect. II. For completeness we give all pertinent definitions. 
and Lemma II.2a is proved.

b) follows from Lemma B.1, 2b, 3 and Hadamard's inequality.

c) follows from Lemma B.2b and B.3 using Hadamard's inequality and Lemma B.1.

Lemma A.2. Let $T_{j i}^{\lambda k}$ be given by $(A .3)$ with $\alpha_{l}=\beta_{l}=l$. Then

$$
\begin{gathered}
\left\|T_{j i}^{\lambda k}\right\| \leqq c\left(\frac{c}{\pi}\right)^{\left(m_{\imath}+m_{j}\right) / 2} \rho\left(s_{j}\right)^{m_{j} / 2} \rho\left(s_{i}\right)^{m_{l} / 2}\left(m_{i} ! m_{j} !\right)^{-1 / 2} \\
\cdot\left(m_{i}-k+1\right)^{\left(m_{t}-k+1\right) / 4}\left(m_{j}-k+1\right)^{\left(m_{j}-k+1\right) / 4},
\end{gathered}
$$

where $c=1+c_{1}+c_{3}, c_{1}$ and $c_{3}$ given by Lemmas $B .2$ and $B .3$ respectively and $\rho(s)=\left(1+c_{1}+c_{2}\right)^{2}, c_{2}$ given by Lemma B.2.

Proof. Assume $k$ even (an analogous argument works if $k$ is odd) and write $T_{j i}^{\lambda k}=W_{j}^{\lambda k} R_{j i}^{\lambda k} V_{i}^{\lambda k}$, where $W_{j}^{\lambda k}$ and $V_{i}^{\lambda k}$ are multiplication operators by the functions

and

$$
(2 \pi)^{-m_{J} / 2}\left(m_{j} !\right)^{-1 / 2} \operatorname{Pf} B_{\lambda}\left(\left(-p^{j}\right)_{k+1, m_{J}}\right)
$$

$$
(2 \pi)^{-m_{1} / 2}\left(m_{i} !\right)^{-1 / 2} \operatorname{Pf} B_{\lambda}^{x}\left(\phi \mid\left(p^{i}\right)_{k+1, m_{2}}\right),
$$

respectively, and $R_{j i}^{\lambda k}: L^{2}\left(R^{m_{i}}\right) \rightarrow L^{2}\left(R^{m_{J}}\right)$ is given by

$$
\begin{aligned}
& R_{j i}^{\lambda k}: f\left(\left(p^{i}\right)_{1, m_{i}}\right) \rightarrow \exp \left\{-\frac{1}{2} \sum_{l=1}^{m_{i}}\left(\omega_{\lambda}\left(p_{l}^{j}\right) s_{j}+i p_{l}^{j} x_{j}\right\} \cdot \int \prod_{r=1}^{k} m_{+}^{\lambda}\left(-p_{r}^{j}, p_{r}^{i}\right)\right. \\
& \cdot \exp \left\{-\frac{1}{2} \sum_{l=1}^{m_{l}}\left(\omega_{\lambda}\left(p_{l}^{i}\right) s_{i}+i p_{l}^{i} x_{i}\right)\right\} \cdot f\left(\left(p^{i}\right)_{1, m_{i}}\right) d^{m_{i}} p^{i},
\end{aligned}
$$

where the symbolic integral is to be interpreted as in Sect. II.

Taking into áccount the form of $m_{+}^{\lambda}$ we write $R_{j i}^{\lambda k}$ as the sum of a product of operators as

$$
R_{j i}^{\lambda k}=\sum_{\mathbf{r}} A_{\mathbf{r}^{\prime}}^{\lambda}\left(s_{j}, x_{j}\right) H_{\lambda}^{k} B_{\mathbf{r}}^{\lambda}\left(s_{i}, x_{i}\right)
$$

where $\mathbf{r}^{\prime}=\left(r_{1}^{\prime}, r_{2}^{\prime} \ldots r_{k}^{\prime}\right), \mathbf{r}=\left(r_{1}, \ldots, r_{k}\right), r_{n}= \pm, r_{n}^{\prime}=\mp, 1 \leqq n \leqq k$, and $\sum_{\mathbf{r}}$ is the sum over all $2^{k}$ sequences $\mathbf{r} . A_{\mathbf{r}}\left(s_{j}, x_{j}\right)$ is the multiplication operator

$$
\prod_{s=1}^{k} \phi_{r_{s} s_{s}}^{\lambda}\left(p_{s}^{j}\right) \exp \left\{-\frac{1}{2} \omega_{\lambda}\left(p_{s}^{j}\right) s_{j}+i p_{s}^{j} x_{j}\right\} \exp \left\{-\frac{1}{2} \sum_{l=k+1}^{m_{j}}\left(\omega_{\lambda}\left(p_{l}^{j}\right) s_{j}+i p_{l}^{j} x_{j}\right)\right\}
$$

$B_{\mathbf{r}}\left(s_{i}, x_{i}\right)$ is the multiplication operator

$$
\prod_{t=1}^{k} \Phi_{r_{t}}^{\lambda}\left(p_{t}^{i}\right) \exp \left\{-\frac{1}{2} \omega_{\lambda}\left(p_{t}^{i}\right) s_{i}+i p_{t}^{i} x_{i}\right\} \exp \left\{-\frac{1}{2} \sum_{l=k+1}^{m_{l}}\left(\omega_{\lambda}\left(p_{l}^{i}\right) s_{i}+i p_{l}^{i} x_{i}\right)\right\}
$$

and $H_{\lambda}^{k}$ is the product of $k$ scaled Hilbert transforms $H_{\lambda}$ of Appendix $\mathrm{C}$ in the first $k$ variables. Thus $\left\|R_{j i}^{\lambda k}\right\| \leqq \sum_{\mathbf{r}}\left\|A_{\mathbf{r}^{\prime}}^{\lambda}\right\|\left\|B_{\mathbf{r}}^{\lambda}\right\|$, where we have used $\left\|H_{\lambda}^{k}\right\| \leqq 1$. 
Let $K(\lambda)$ be a smooth, monotone decreasing function of $\lambda \in[0,1]$ such that $K(1)>0, \inf _{\lambda \in[0.1]}\left(-K^{\prime}(\lambda)\right)>0$ and $K(0)=K_{c}$, where $K_{c}$ is the unique solution of $e^{2 K}=\operatorname{coth} K$. Let $K^{*}$ and $\varepsilon(k) \geqq 0$ be defined implicity by $e^{2 K^{*}}=\operatorname{coth} K$ and $\cosh \varepsilon(k)=\cosh 2\left(K^{*}-K\right)+\sinh 2 K^{*} \cdot \sinh 2 K(1-\cos k)$. Let $\bar{m}=\inf _{\lambda \in(0,1]} 2\left(K^{*}-\right.$ $K) / \lambda \geqq 4 \inf _{\lambda \in(0,1]}\left(-K^{\prime}(\lambda)\right)>0$ and $-4 K^{\prime}(0) \equiv m>0$. All constants appearing in the subsequent lemmas depend only on the choice of the function $K(\lambda)$.

Lemma B.1 Let $\omega_{\lambda}(k) \equiv \varepsilon(\lambda k) / \lambda$, then

$$
\inf _{\lambda \in(0,1]} \inf _{|k| \leqq \pi / \lambda} \omega_{\lambda}(k)\left(\bar{m}^{2}+k^{2}\right)^{-1 / 2} \equiv c_{0}>0 .
$$

Proof. $\varepsilon(\lambda k)=\log \left(\eta+\left(\eta^{2}-1\right)^{1 / 2}\right), \quad$ where $\quad \eta(\lambda k)=\cosh 2\left(K^{*}-K\right)+\sinh 2 K^{*}$. $\sinh 2 K(1-\cos \lambda k)$. Clearly, there is a constant $\gamma_{1}$ such that $1 \leqq \eta \leqq \gamma_{1}$. Let

$$
\gamma_{2}=\inf \left\{x^{-1} \log (1+x): 0<x<\left(\gamma_{1}^{2}-1\right)^{1 / 2}\right\} \text {, }
$$

then

$$
\varepsilon(\lambda k) \geqq \gamma_{2}\left(\eta^{2}-1\right)^{1 / 2} \geqq \sqrt{2} \gamma_{2} \cdot(\sinh 2 K(1))^{1 / 2}(1-\cos \lambda k)^{1 / 2} .
$$

Therefore,

$$
\omega_{\lambda}(k) \geqq \gamma_{2} \sqrt{2}(\sinh 2 K(1))^{1 / 2}\left|\inf _{0<x \leqq \pi} x^{-1}(1-\cos x)^{1 / 2}\right||k| \equiv \gamma_{3}|k|\left(\gamma_{3}>0\right) .
$$

Since $\omega_{\lambda}(k) \geqq \bar{m}$, the proof follows at once. Let

and

$$
\begin{aligned}
x_{1} & =\operatorname{coth} K^{*} \operatorname{coth} K, x_{2}=\tanh K^{*} \operatorname{coth} K, \\
\theta_{ \pm}(z) & =\left[\left(1-x_{1}^{-1} z^{ \pm 1}\right)\left(1-x_{2}^{-1} z^{ \pm 1}\right)\right]^{\mp 1 / 2}
\end{aligned}
$$

so that

$$
\theta(z)=\theta_{+}(z) \theta_{-}(z)
$$

$$
\Phi_{ \pm}(z)=\left(x_{1} x_{2}\right)^{ \pm 1 / 2} \theta_{ \pm}^{-1}(z)
$$

Lemma B.2. a) There are positive constants $c_{1}^{\prime}, \ldots, c_{5}^{\prime}$ such that for all $\lambda \in(0,1], 1<c_{1}^{\prime} \leqq x_{1}(\lambda) \leqq c_{2}^{\prime} ; 1 \leqq x_{2}(\lambda) \leqq c_{3}^{\prime} ; c_{4}^{\prime} \leqq \lambda^{-1}\left(x_{2}(\lambda)-1\right) \leqq c_{5}^{\prime}$.

b) $\sup _{\lambda \in(0,1]|k| \leq \pi / \lambda} \sup \left|\lambda^{1 / 2} \theta^{-1}\left(e^{i \lambda k}\right)\right| \equiv c_{1}<\infty$.

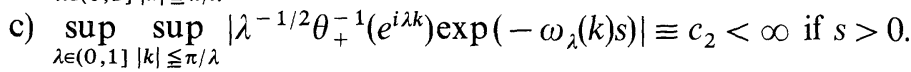

Proof. a) follows directly from the definitions, and b) and c) follow from a). We consider c):

$$
\begin{aligned}
\left|\lambda^{-1 / 2} \theta_{+}^{-1}\left(e^{i \lambda k}\right)\right| & =\left|\frac{\left(x_{1}-e^{i \lambda k}\right)\left(x_{2}-e^{i \lambda k}\right)}{\lambda x_{1} x_{2}}\right|^{1 / 2} \\
& \leqq\left(c_{2}^{\prime}+1\right)\left|\frac{x_{2}-1}{\lambda}+\frac{1-e^{i \lambda k}}{\lambda}\right| \leqq\left(c_{2}^{\prime}+1\right)\left(c_{5}^{\prime}+|k|\right) .
\end{aligned}
$$


The proof is completed by using Lemma B.1.

Let

$$
m_{-}^{\lambda}(k, q)=\lambda e^{i(k+q) \lambda}\left(e^{i(k+q) \lambda}-1\right)^{-1}\left|\theta_{+}^{-1}\left(e^{i \lambda k}\right) \theta^{-1}\left(e^{i \lambda q}\right)-\theta_{+}^{-1}\left(e^{i \lambda q}\right) \theta_{-}^{-1}\left(e^{i \lambda k}\right)\right| .
$$

Lemma B.3. $\sup _{\lambda \in(0,1]} \sup _{|k|,|q|<\pi / \lambda}\left|m_{-}^{\lambda}(k, q)\right| \equiv c_{3}<\infty$.

Proof. Let

$$
\begin{gathered}
n_{-}^{\lambda}(k, q) \equiv \theta_{+}^{-1}\left(e^{i \lambda k}\right) \theta_{-}^{-1}\left(e^{i \lambda q}\right)-\theta_{+}^{-1}\left(e^{i \lambda k}\right) \theta_{-}^{-1}\left(e^{i \lambda k}\right)=\theta_{-}^{-1}\left(e^{i \lambda q}\right) \int_{-q}^{k} \frac{d \theta_{+}^{-1}}{d u}\left(e^{i \lambda u}\right) d u \\
-\theta_{-}^{-1}\left(e^{i \lambda k}\right) \cdot \int_{-k}^{q} \frac{d \theta_{+}^{-1}}{d u}\left(e^{i \lambda u}\right) d u .
\end{gathered}
$$

Now,

$$
\frac{d \theta_{+}^{-1}}{d u}\left(e^{i \lambda u}\right)=\frac{\lambda}{i} e^{i \lambda u} \theta_{-}^{-1}\left(e^{i \lambda u}\right)\left[x_{1}^{-1}\left(1-x_{2}^{-1} e^{i \lambda u}\right)+x_{2}^{-1}\left(1-x_{1}^{-1} e^{i \lambda u}\right)\right] .
$$

From Lemma B.2 it follows that there exists a constant $\alpha_{1}$ such that $\left|n_{-}^{\lambda}(k, q)\right| \leqq$ $\alpha_{1}|k+q|$, hence $\left|m_{-}^{\lambda}(k, q)\right| \leqq \alpha_{1} /\left|\frac{e^{i \lambda(k+q)}-1}{\lambda(k+q)}\right|$. Let $\alpha_{2}=\inf _{0<|x| \leqq \pi}\left|\left(e^{i x}-1\right) / x\right|>0$. Thus, $m_{-}^{\lambda}(k, q) \leqq \frac{\alpha_{1}}{\alpha_{2}}$ if $|k+q| \lambda \leqq \pi$. To handle the region $\pi \leqq \lambda|k+q| \leqq 2 \pi$, note that $n_{-}^{\lambda}(k, q)$ can also be written as

$$
\begin{aligned}
n_{-}^{\lambda}(k, q)= & \theta^{-1}\left(e^{i \lambda q}\right)-\theta^{-1}\left(e^{i \lambda k}\right)+\theta_{-}^{-1}\left(e^{i \lambda q}\right) \int_{q}^{k} \frac{d \theta_{+}^{-1}}{d u}\left(e^{i \lambda u}\right) d u-\theta_{-}^{-1}\left(e^{i \lambda k}\right) \\
& \cdot \int_{k}^{q} \frac{d \theta_{+}^{-1}}{d u}\left(e^{i \lambda u}\right) d u .
\end{aligned}
$$

By direct computation, using Lemma B.2, we can show that $\frac{d \theta^{-1}}{d u}\left(e^{i \lambda u}\right)$ is uniformly bounded in $\lambda$. Therefore, there exists a constant $\alpha_{3}$ such that $\mid m_{-}^{\lambda}$ $(k, q)\left|\leqq \alpha_{3}\right| k-q|\lambda /| e^{i \lambda(k+q)}-1 \mid$. Assume $\pi \leqq \lambda(k+q) \leqq 2 \pi$ and let $\varepsilon_{1}=\pi-\lambda k, \varepsilon_{2}=$ $\pi-\lambda q$. Since $\lambda k, \lambda q \leqq \pi, \varepsilon_{i} \geqq 0(i=1,2)$. Also, $\varepsilon_{1}+\varepsilon_{2} \leqq \pi$, hence

$$
\left|m_{-}^{\lambda}(k, q)\right| \leqq \alpha_{3}\left|\varepsilon_{1}-\varepsilon_{2}\right| /\left|e^{-i\left(\varepsilon_{1}+\varepsilon_{2}\right)}-1\right| \leqq \alpha_{3} /\left|\left(e^{i\left(\varepsilon_{1}+\varepsilon_{2}\right)}-1\right) /\left(\varepsilon_{1}+\varepsilon_{2}\right)\right| \leqq \alpha_{3} / \alpha_{2} .
$$

Similar considerations hold for the region $-2 \pi \leqq \lambda(k+q) \leqq-\pi$ and the proof is complete.

Lemma B.4. Let $\omega(p) \equiv\left(p^{2}+m^{2}\right)^{1 / 2}$. Then we have the following pointwise convergence as $\lambda \rightarrow 0$ :
a) $m_{-}^{\lambda}(p, q) \rightarrow-(p-i m)^{-1 / 2}(q-i m)^{-1 / 2}(\omega(p)-\omega(q)) / i(p+q)$,
b) $\Phi_{-}^{\lambda}(p) \rightarrow(m+i p)^{-1 / 2}$,
c) $\Phi_{+}^{\lambda}(p) \rightarrow(m-i p)^{1 / 2}$
d) $\omega_{\lambda}(p) \rightarrow \omega(p)$.

Proof. Follows from the definitions. 


\section{Appendix C}

The scaled Hilbert transforms, $H_{\lambda}, \lambda \in[0,1]$, used in the proof of Theorem II.1, are defined as the closure of the operators associated with the forms

$$
t_{\lambda}(g, f)=\int_{-\pi / \lambda}^{\pi / \lambda} \bar{g}(k)\left[\frac{P}{\pi} \int_{-\pi / \lambda}^{\pi / \lambda} \frac{-i \lambda}{\left(1-e^{i \lambda(k-q)}\right)} f(q) d q\right] d k, \quad \lambda \in(0,1],
$$

where $g, f \in C_{0}^{\infty}(R) . t_{0}$ is the form associated with the Hilbert transform $H_{0}$ on the line. We have

Lemma C.1. Let $\chi_{\lambda} \equiv \chi_{[-\pi / \lambda, \pi / \lambda]}$, the characteristic function of $\left[-\frac{\pi}{\lambda}, \frac{\pi}{\lambda}\right]$. Then

a) $\left|t_{\lambda}(g, f)\right| \leqq|f|_{L^{2}(R)}|g|_{L^{2}(R)}$; the associated operator, $H_{\lambda}$, satisfies $H_{\lambda}=\chi_{\lambda} H_{\lambda} \chi_{\lambda}$, $\left|H_{\lambda}\right|_{L^{2} \rightarrow L^{2}} \leqq 1$ and extends by continuity to $L^{2}(R)$.

b) $H_{\lambda} \stackrel{\lambda \rightarrow 0}{\rightarrow} H_{0}$ in the strong $L^{2}$ operator sense.

Proof. a) follows by the change of variables $u=\lambda q, v=\lambda k$ and the fact that the norm of the Hilbert transform on $[-\pi, \pi]$ is one.

b) By a) it is enough to show the result for a dense set. Suppose $f \in C_{0}^{\infty}$. Then

$$
\left|\left(H_{\lambda}-H_{0}\right) f\right|_{L^{2}(R)} \leqq\left|\left(H_{\lambda}-\chi_{\lambda} H_{0}\right) f\right|_{L^{2}}+\left|\left(I-\chi_{\lambda}\right) H_{0} f\right|_{L^{2}}
$$

so that the second term goes to zero. Suppose that supp $f \subset\left\{q:|q|<\pi / \lambda_{0}\right\}$, then for all $\lambda<\lambda_{0}$

$$
\left|\left(H_{\lambda}-\chi_{\lambda} H_{0}\right) f\right|_{L^{\infty}(R)} \leqq \sup _{|q|<\pi / \lambda_{0}, k \mid \leqq \pi / \lambda .}\left|i \lambda\left(e^{i \lambda(k-q)}-1\right)^{-1}-(k-q)^{-1}\right||f|_{L^{1}(R)} .
$$

For $\lambda<\lambda_{0} / 2$ the sup can be taken over the set $\lambda|k-q|<3 \pi / 2$; but

$$
\begin{aligned}
\left|i \lambda\left(e^{i \lambda x}-1\right)^{-1}-x^{-1}\right| & =\left|\left(\int_{0}^{\lambda}(\lambda-t)\left(-x^{2} e^{i t x}\right) d t\right) /(2 x \sin (\lambda x / 2))\right| \\
& \leqq(\lambda / 2)\left|\left(\sin \left(\frac{\lambda x}{2}\right) /(\lambda x / 2)\right)\right|^{-1} \leqq 2 \pi \lambda / 4 \sqrt{2}
\end{aligned}
$$

for $\lambda|x| \leqq 3 \pi / 2$, since $y^{-1} \sin y>2 \sqrt{2} / 3 \pi$ for $y<3 \pi / 4$, so that for all $\lambda<\lambda_{0} / 2$, $\left|\left(H_{\lambda}-\chi_{\lambda} H_{0}\right) f\right|_{L^{\infty}(R)} \leqq c \lambda|f|_{L^{1}(R)}$. Thus

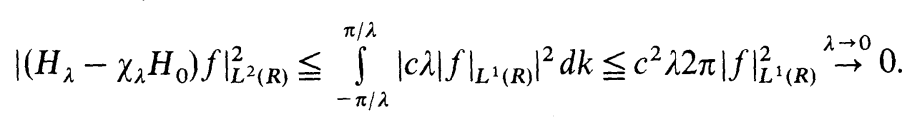

For $f \in C_{0}^{\infty}(R)$, define the approximate rapidity transform, $H_{\varepsilon}^{R}, \varepsilon>0$, by

$$
\left(H_{\varepsilon}^{R} f\right)(x)=(2 \pi i)^{-1} \int_{-\infty}^{\infty}\left[(\sinh (x-y)+i \varepsilon)^{-1}+(\sinh (x-y)-i \varepsilon)^{-1}\right] f(y) d y
$$

and the rapidity transform, $H_{0}^{R}$, by taking the principal value integral in the above.

Lemma C.2. a) For $\varepsilon \in[0,1), H_{\varepsilon}^{R}$ extends to a bounded operator on $L^{2}$ and $\left\|H_{\varepsilon}^{R}\right\| \leqq\left(1-\varepsilon^{2}\right)^{-1 / 2}$.

b) $H_{\varepsilon}^{R} \stackrel{s}{\rightarrow} H_{0}^{R}$ as $\varepsilon \rightarrow 0$. 
Proof. a) By contour integration the Fourier transform, $\hat{H}_{\varepsilon}^{R}$, of $H_{\varepsilon}^{R}$ is found to be $\hat{H}_{\varepsilon}^{R}(k)=\left(1-\varepsilon^{2}\right)^{-1 / 2} \sinh k\left(\pi / 2-\sin ^{-1} \varepsilon\right)(\cosh (k \pi / 2))^{-1}, \quad \varepsilon \in[0,1)$. Thus $\left|\hat{H}_{\varepsilon}^{R}\right|_{L^{\infty}} \leqq\left(1-\varepsilon^{2}\right)^{-1 / 2}$.

b) follows from a) and the pointwise convergence $\hat{H}_{\varepsilon}^{R}(k) \rightarrow \hat{H}_{0}^{R}(k)$, as $\varepsilon \rightarrow 0$.

\section{Appendix D}

We will need the following lemma in the proof of Theorem IV.1:

Lemma D.1 Let $\Delta_{k} T_{j i}\left(s_{j}, s_{i}\right)=T_{j i}\left(s_{j}+b \delta_{j k}, s_{i}+b \delta_{i k}\right)-T_{j i}\left(s_{j}, s_{i}\right), k=i, j$ and $D_{k} T_{j i}$ the operator defined by taking the derivative $\partial / \partial s_{k}$ inside the integral defining $T_{j i}$ of Sect. II. Then for $0<\delta<s_{i}, s_{j}$ and some $M_{\delta}, 0<M_{\delta}<\infty$,

with

$$
b^{-1} \Delta_{k} T_{j i}\left(s_{j}, s_{i}\right)=D_{k} T_{j i}\left(s_{j}, s_{i}\right)+R_{k},
$$

and

$$
\mid D_{k} T_{j i}\left(s_{j}, s_{i} \mid \leqq M_{\delta} c_{j i}\left(s_{j}-\delta_{j k} \delta, s_{i}-\delta_{i k} \delta\right)\right.
$$

$$
\left|R_{k}\right| \leqq(b / 2) M_{\delta} \cdot c_{j i}\left(s_{j}-\delta_{j k} \delta, s_{i}-\delta_{k i} \delta\right)
$$

where $c_{j i}$ is the right side of Lemma 11.2a.

Proof. We give the proof for $k=j$, the case $k=i$ being similar. By the decomposition (A.4) of $T_{j i}^{\lambda}$ and the decomposition $T_{j i}^{\lambda k}=W_{j}^{\lambda k} R_{j i}^{\lambda k} V_{i}^{\lambda k}$ of Lemma A.2 it is sufficient to consider the term $R_{j i}^{\lambda k}$ given in (A.3). A typical term of $R_{j i}^{\lambda k}$ can be written, for $\lambda=0$ and suppressing the $\lambda=0$ index, $b^{-1}\left(A_{\mathbf{r}^{\prime}}\left(s_{j}+b\right)-A_{\mathbf{r}^{\prime}}\left(s_{j}\right)\right) H^{k} B_{\mathbf{r}}\left(s_{i}\right)$. Using Taylor's theorem, with $b>0$, we obtain

$$
\begin{aligned}
b^{-1}\left(A_{\mathbf{r}^{\prime}}\left(s_{j}+b\right)-A_{\mathbf{r}^{\prime}}\left(s_{j}\right)\right)= & \left(-\frac{1}{2} \sum_{l=1}^{m_{j}} \omega\left(p_{l}^{j}\right)\right) A_{\mathbf{r}^{\prime}}\left(s_{j}\right)+b^{-1} \int_{s_{j}}^{s_{j}+b}\left(s_{j}+b-t\right) \\
& \cdot\left(-\frac{\sum \omega}{2}\right)^{2} A_{\mathbf{r}^{\prime}}(t) d t .
\end{aligned}
$$

For any $\delta, 0<\delta<s_{j}, t \in\left[s_{j}, s_{j}+b\right], u e^{-s_{\jmath} u}$ and $u^{2} e^{-s_{\jmath} u}$ are bounded by $M_{\delta} e^{-\left(s_{j}-\delta\right) u}$, $0<M_{\delta}<\infty$, so that with $u=\sum \omega$ we have the bound

$$
\left|b^{-1}\left(A_{\mathbf{r}^{\prime}}\left(s_{j}+b\right)-A_{\mathbf{r}^{\prime}}\left(s_{j}\right)\right)\right| \leqq M_{\delta}\left|A_{\mathbf{r}^{\prime}}\left(s_{j}-\delta\right)\right|+(b / 2) M_{\delta}\left|A_{\mathbf{r}^{\prime}}\left(s_{j}-\delta\right)\right| .
$$

The case $b<0$ is treated similarly and the result follows from Lemma A.2 and its proof.

Proof of Theorem $I V .1$. We first justify the passage of the derivatives through the infinite sum and integrals of (2.2). Consider, for example, the derivative $\partial S_{2 N}^{L} / \partial s_{r}$, $1<r<2 N$, where $S_{2 N}^{L}$ is given by Lemma II.1. We have, suppressing inessential arguments in the functions for notational simplicity and abbreviating the sum by $\sum^{\infty}$

$$
b^{-1}\left(S_{2 N}^{L}\left(s_{r}+b\right)-S_{2 N}^{L}\left(s_{r}\right)\right)=\sum_{l=2 N-1}^{\infty} \prod_{m_{l+1} m_{l}}^{r}\left\{b^{-1} \Delta_{r} T_{m_{r+1} m_{r}} \Delta_{r} T_{m_{r} m_{r-1}}\right.
$$




$$
\begin{array}{ll}
\left.+b^{-1} \Delta_{r} T_{m_{r+1} m_{r}} T_{m_{r} m_{r-1}}+T_{m_{r+1} m_{r}} b^{-1} \Delta_{r} T_{m_{r} m_{r-1}}\right\} \\
\cdot \prod_{k=r-2}^{1} T_{m_{k+1} m_{k}} \theta_{m_{1}}\left(x_{1}, s_{1}\right), & \text { (D. } 1)
\end{array}
$$

where $\Delta_{k} T_{j i}$ is given by Lemma D.1. Substituting for $\Delta_{r} T_{m_{r+1} m_{r}}$, using Lemma D.1, we see that the right side of (D.1) is equal to the sum of two absolutely convergent series. The first series is independent of $b$ and is given by the series for $S_{2 N}^{L}$ with $D_{r} T_{m_{r} m_{r-1}}$ and $D_{r} T_{m_{r+1} m_{r}}$ replacing $T_{m_{r} m_{r-1}}$ and $T_{m_{r+1} m_{r}}$, respectively. The absolute convergence follows from the bounds in Lemma D.1 and Lemma II.2. The second series is bounded by $b$ times an absolutely convergent series with bound independent of $b$ again using Lemma D.1 for the remainder terms and Lemma II.2. Thus the $b \rightarrow 0$ limit of (D.1) exists and is given by the series obtained by differentiating the series for $S_{2 N}^{L}$ term by term inside the integral.

We now write the generic term, call it $G_{0}$, of $\sum_{i=1}^{k-1}\left(x_{i} \partial S_{k}^{L} / \partial s_{i}-s_{i} \partial S_{k} / \partial x_{i}\right)$ in terms of rapidity variables and let $G_{\varepsilon}, \varepsilon \in[0,1]$, denote $G_{0}$ with terms of the form $\left(\omega\left(p_{i}\right)+\omega\left(p_{j}\right)\right) /\left(p_{i}-p_{j}\right)=\operatorname{coth}\left(\theta_{i}-\theta_{j}\right)$ replaced by

$$
\pi i\left(\cosh \theta_{i} H_{\varepsilon}^{R}\left(\theta_{i}, \theta_{j}\right) \cosh \theta_{j}-\sinh \theta_{1} H_{\varepsilon}^{R}\left(\theta_{i}, \theta_{j}\right) \sinh \theta_{j}\right),
$$

where $H_{\varepsilon}^{R}\left(\theta_{i}, \theta_{j}\right)$ is the kernel of the operator of Lemma C.2. By following the proof of Lemma II.6 in Appendix A, boundedness (uniform in $\varepsilon$ ) of $G_{\varepsilon}$ follows by using Lemma C.2a. By using $H_{\varepsilon}^{R} \stackrel{s}{\rightarrow} H_{0}^{R}$ (by Lemma C.2b) in place of $H_{\lambda}^{k} \stackrel{s}{\rightarrow} H_{0}^{k}$ we have $\lim _{\varepsilon \rightarrow 0} G_{\varepsilon}=G_{0} . G_{\varepsilon}$ can be written in the form

where

$$
G_{\varepsilon}=\int J_{n}\left(\boldsymbol{\theta}_{n}\right)\left(\mathbf{n} \cdot \nabla_{\theta} e^{K_{n}}\right) d \boldsymbol{\theta}_{n}
$$

$$
K_{n}=m\left(\sum_{i=1}^{k-1}\left(-s_{i} \sum_{j=1}^{n_{j}} \cosh 2 \theta_{j}^{(i)}+i x_{i} \sum_{j=1}^{n_{j}} \sinh 2 \theta_{j}^{(i)}\right)\right.
$$

$\nabla_{\theta}$ and $\mathbf{n}$ are now the $\sum_{i=1}^{k} n_{i}$ dimensional vectors of Sect. IV, $n$ is a multi-index and $J_{n}$ is a $C^{\infty}$ function of $\tanh \left(\theta_{i}-\theta_{j}\right), \cosh \left(\theta_{i}-\theta_{j}\right)$ and $\sinh \left(\theta_{i}-\theta_{j}\right)$, i.e. of the difference variables. By integrating by parts $G_{\varepsilon}=0$ which implies $G_{0}=0$ and the result follows.

\section{References}

1. Schultz, T. D., Mattis, D. C., Lieb, E. H.: Rev. Mod. Phys. 36, 856 (1964)

2. Abraham, D.: Phys. Letts. 61A, 271 (1977); I. Commun. Math. Phys. 59, 17-34 (1978); II. Commun. Math. Phys. 60, 181-191 (1978); III. Commun. Math. Phys. 60, 205-213 (1978)

3. Schor, R. S., O'Carroll, M. L.: "The quantum field theory associated with the infinite Lattice twodimensional Ising Model," Pontificia Universidade Catolica do Rio de Janeiro (PUC/RJ), Department of Mathematics preprint, Dec. 1979

4. Berezin, F. A.: The method of second quantization. New York: Academic Press 1966

5. O'Carroll, M. L., Schor, R. S.: "The correlation functions and scaling limit of the two-dimensional ising model," PUC/RJ, Department of Mathematics preprint, April 1980

6. Glimm, J., Jaffe, A.: Phys. Rev. Letts. 33, 7 p. 440-442, (1974) 
7. Osterwalder, K., Schrader, R.: Commun. Math. Phys. 31, 83 (1973); Commun. Math. Phys. 42, 281 (1975); Constructive quantum field theory, ed. Velo, G., Wightman, A. Berlin: Springer 1973

8. Mc Coy, B. M., Wu, T. T., Tracy, C. A.: Phys. Rev. Lett. 38, 793 (1977)

9. Sato, M., Miwa, T., Jimbo, M.: Proc. Jpn. Acad. 53, Ser. A, 6-10 (1977)

10. Simon, B.: The $P(\phi)_{2}$ Euclidean (quantum) field theory. Princeton, N.J.: Princeton University Press 1974

Communicated by K. Osterwalder

Received April 23, 1981; in revised form July 20, 1981 